\title{
Endodontic Management of Mandibular Second Molar with a Type II Vertucci Canal Configuration Evaluated Using Cone- Beam Computed Tomography Scanning - A Case Report.
}

\author{
Rajeesh M K ${ }^{1}$, Gokulraj ${ }^{2}$, Rakhee Ramdas ${ }^{3}$, Nadira K Rehman ${ }^{4}$, \\ Muhammed Abdul Rehman \\ ${ }^{I}$ (Department of conservative dentistry and endodontics, Kozhikode govt dental College, India.) \\ ${ }^{2}$ (Department of conservative dentistry and endodontics, Kozhikode govt dental College, India.) \\ ${ }_{3}^{3}$ (Department of conservative dentistry and endodontics, Kozhikode govt dental College, India.) \\ ${ }_{5}^{4}$ (Department of conservative dentistry and endodontics, Kozhikode govt dental College, India.) \\ ${ }^{5}$ (Department of conservative dentistry and endodontics, Kozhikode govt dental College, India.)
}

\begin{abstract}
The variability of root canal morphology of multirooted teeth represents a continuous challenge to endodontic diagnosis and therapeutics. This report extends the range of known possible anatomical variations to include teeth with lesser number of roots with varied root canal morphology. Variations of root canal systems need not always be in the form of extra canals. Clinicians should be aware that there is a possibility of existence of fewer number of roots with varied root canal anatomy than the normal root canal anatomy. Thus the aim of this article is to present a case report of endodontically managed mandibular second molar with a Vertucci type II canal configuration.
\end{abstract}

Keywords: endodontic diagnosis, root canal morphology, Vertucci type II canal configuration

\section{Introduction:}

Successful root canal treatment depends on adequate debridement and filling of the entire root canal system, but the relative simplicity and uniformity of external surfaces of roots often masks their internal complexicity(1). According to Vertucci, the mandibular second molar is similar to the first, except that the roots are shorter, the canals more curved, and the range of the variations broader. Very often (64\%) the mesial root has two canals, approximately $38 \%$ incidence for type II and $26 \%$ incidence for type IV. In the distal root, there is almost always only one independent canal (92\%) (type I), rarely type II (3\%) or type IV (4\%). When type I is a single canal extends from the pulp chamber to the apex; type II are two separate canals leave the pulp chamber and join short of the apex to form one canal and the type IV are two separate and distinct canals extend from the pulp chamber to the apex(2). Mandibular second molars usually have two roots and three root canals but variations in the number of roots as well as canal morphology are not uncommon. Which includes single canal, two canals, three and four canals, five canals(3).

Cleaning and shaping root canal systems are essential steps in root-canal treatment. The goal of root canal treatment is to clean the root canal systems as thoroughly as possible and to fill it in all its dimensions. In depth knowledge of the root canal anatomy of each tooth is crucial in order to reach this goal. Thus, it is necessary for the clinician to have knowledge of dental anatomy and its variations(4).

\section{Case Report:}

A 45-year old female patient reported to the department of conservative dentistry and endodontics with the chief complaint of "spontaneous toothache in her left lower back tooth region for two days". The pain intensifie by thermal stimuli and on mastication. History revealed intermittent pain in the same tooth with hot and cold stimuli for the past one month. The patient's medical history was non-contributory. Clinical examination revealed a carious mandibular left first and second molar (tooth \#36 and 37), which was tender to percussion. Palpation of the buccal and lingual soft tissue of the tooth did not reveal any tenderness. The tooth was not mobile and periodontal probing around the tooth was within physiological limits. Vitality testing of the involved tooth with heated gutta-percha (Dentsply Maillefer, Ballaigues, Switzerland) and endofrost cold pulp tester (coltene/whaledent Gmbh+Co, KGRaiffeisenstr, Germany) caused an intense lingering pain, whereas electronic pulp stimulation (Parkell Electronics, Farmingdale, NY, USA) caused an early response. A preoperative radiograph revealed a temprorary filling with IRM was done from some private clinic, which was approaching the pulp space (Fig-1a). From the clinical, radiographic and sensibility test findings, a diagnosis of symptomatic irreversible pulpitis with symptomatic apical periodontitis was made and endodontic treatment was suggested to the patient in both tooth \#36 and \#37. 
Radiographic evaluation of the involved tooth indicates a variation in the canal anatomy (Fig- 1a). The tooth was anesthetized with 2\% lignocaine containing 1:100,000 epinephrine (Xylocaine; AstraZeneca Pharma Ind Ltd, Bangalore, India) followed by rubber dam isolation. An endodontic access cavity was established. Clinical examination with a DG-16 endodontic explorer (Hu-Friedy, Chicago, TL) revealed only two canal openings, one in mesial and one in distal root. Examination of the pulp chmber floor was done under Dental Operating Microscope (DOM) (Seiler Revelation, St Louis, MO), no other canal was identified. Coronal enlargement was done with a nickel-titanium ProTaper series orifice shaper (Dentsply Maillefer, Ballaigues, Switzerland) to improve the straight line access (Fig-1b). The working length was determined with the help of an apex locator (Root ZX; Morita, Tokyo, Japan) and later confirmed using a radiograph. (Fig1c).

To confirm this unusual morphology, it was decided to perform CBCT imaging of the tooth. Access cavity was sealed with IRM cement (Dentsply De Trey GmbH, Konstanz, Germany). An informed consent was obtained from the patient, and a multislice CBCT scan of left quadrant of the mandible was performed (Carestream Health Inc, Rochester,NY) with a tube voltage of $60-90 \mathrm{KV}$ and a tube current of 2-5mA. The involved tooth was focused, and the morphology was obtained in coronal, axial, and sagittal sections of 0.09$0.5 \mathrm{~mm}$ thickness. CBCT scan slices revealed a type II Vertucci canal configuration(Two separate canals leave the pulp chamber and join short of the apex to form one canal) (Fig-2).

CBCT images provided valuable information regarding the root canal configuration and confirmed the presence of type II Vertucci canal configuration in tooth \#37. At the second appointment, the patient was asymptomatic. After administering local anaesthesia, cleaning and shaping was performed under rubber dam isolation using PT nickel-titanium rotary instruments with a standard shaping and finishing files. Irrigation was performed using normal saline, 2.5\% sodium hypochloride solution and 17\% EDTA and 2\% chlorhexidine digluconate was used as the final irrigant. The canals were dried with absorbent points (Dentsply Maillefer) and obturation was performed using warm vertical compaction of gutta-percha (Dentsply Maillefer) and AH Plus resin sealer (Maillefer, Dentsply, Konstanz, Germany) (Fig-3(a-b)). The tooth was then restored with a posterior composite resin core (3M ESPE FILTEK Z350 XT; 3M Dental products, St Paul, MN). The patient was advised a full coverage porcelain crown and was asymptomatic during the follow up period of 6 months.

\section{Discussion:}

When anatomic variations are detected clinically, treatment can be performed with conventional or rotary instrumentation and obturation techniques respecting technical and biological principles. Endodontic success in teeth with variations in the number and morphology of canals requires a correct diagnosis and careful clinical and radiographic inspection(5,6). The amount of information gained from conventional radiographs is limited by the fact that three-dimensional anatomy of the area being radiographed is compressed into a two-dimensional image. CBCT scanning has been used in endodontics for the effective evaluation of the root canal morphology. The present case confirms the necessity for meticulous examination of the pulpal floor at high magnification under sufficient illumination of the DOM and emphasize the importance of CBCT in intraoperative assessment(7).

In the present case, CBCT scanning was used for a better understanding of the complex root canal anatomy. CBCT axial and sagittal images confirmed the presence of Vertucci type II canal pattern (i.e. two canal orifices join together and exit as one apical foramen) in a mandibular second molar. Thus the role of dental operating microscope and CBCT scanning was pivotal in the diagnosis of this unusual root canal system and towards its successful endodontic management.

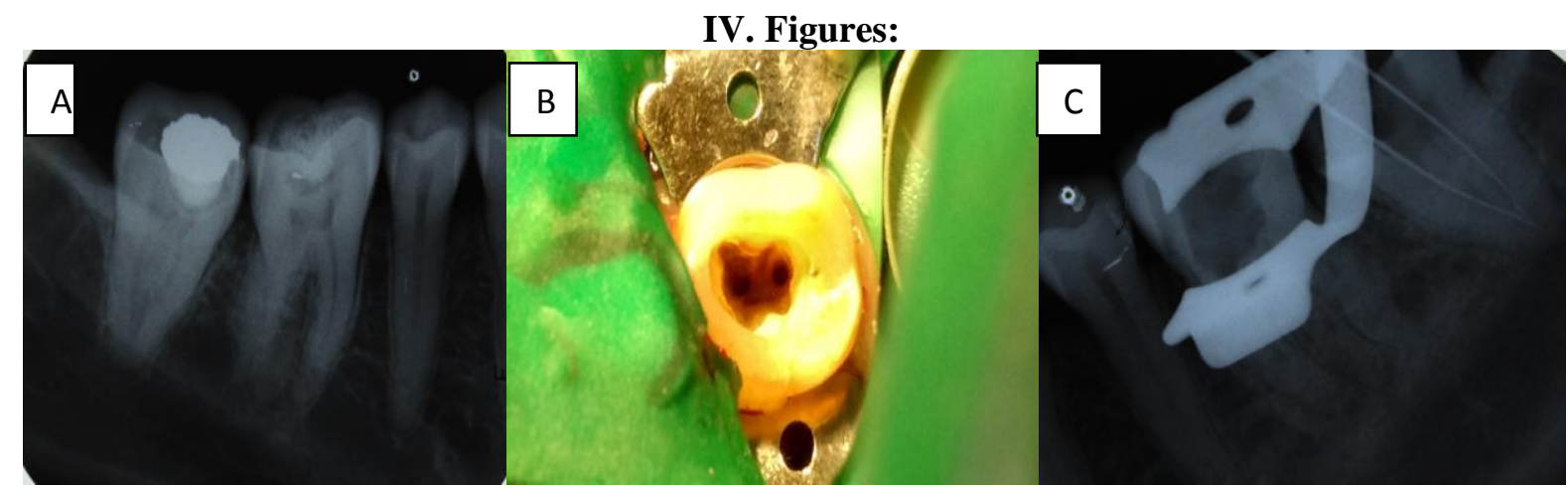

[Figure-1]: (a) Preoperative intra oral periapical radiograph of tooth \#37 and 36. (b) Access opening showing two canal orifice one mesial and one distal (c) working length determination of tooth \#37. 


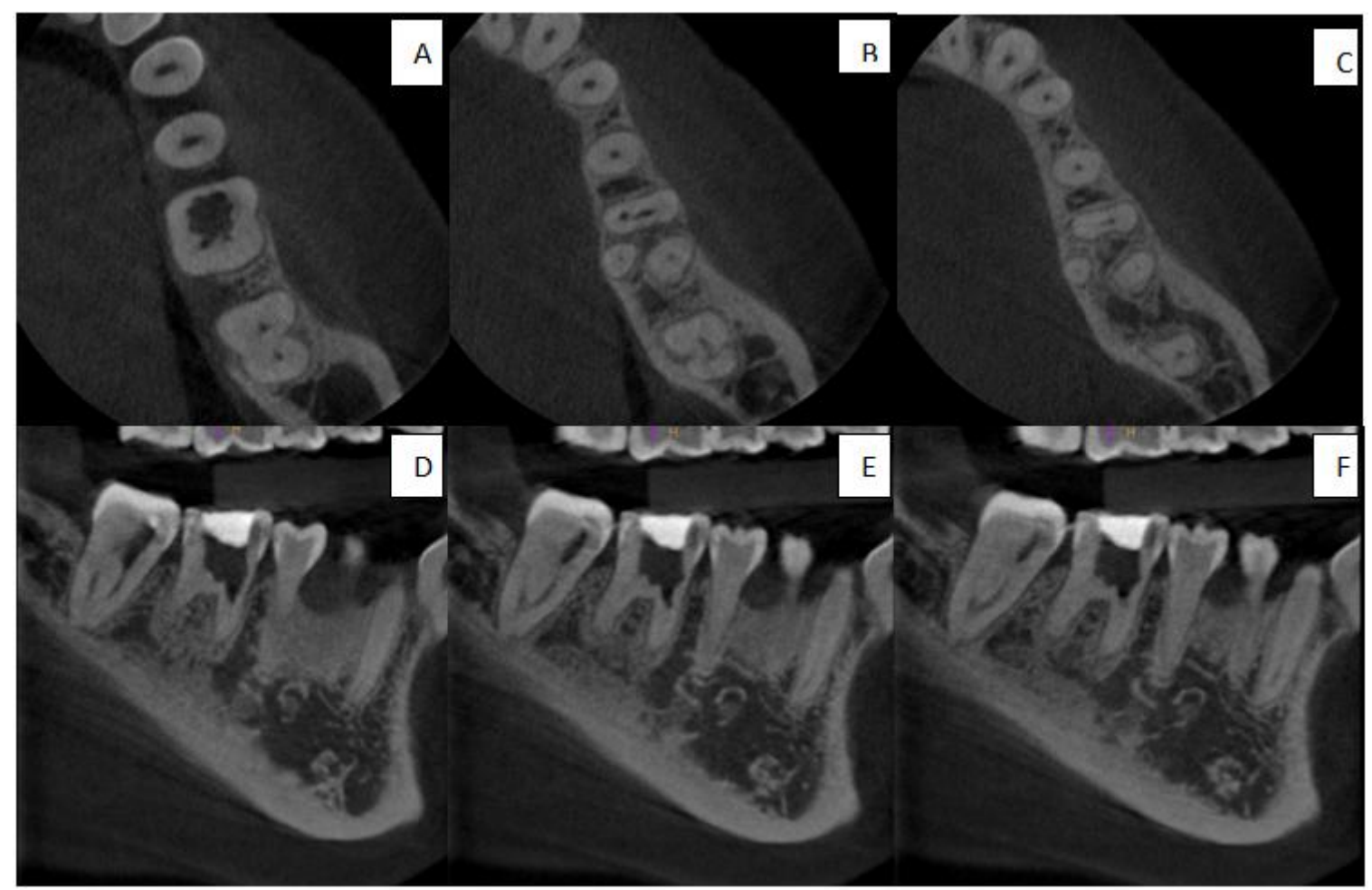

[Figure-2]; (a-c) -axial image showing two canal orifice join together and exit as one apical foramen, (d-f)sagittal sections showing the joining of two ccanals as one as it goes apically.

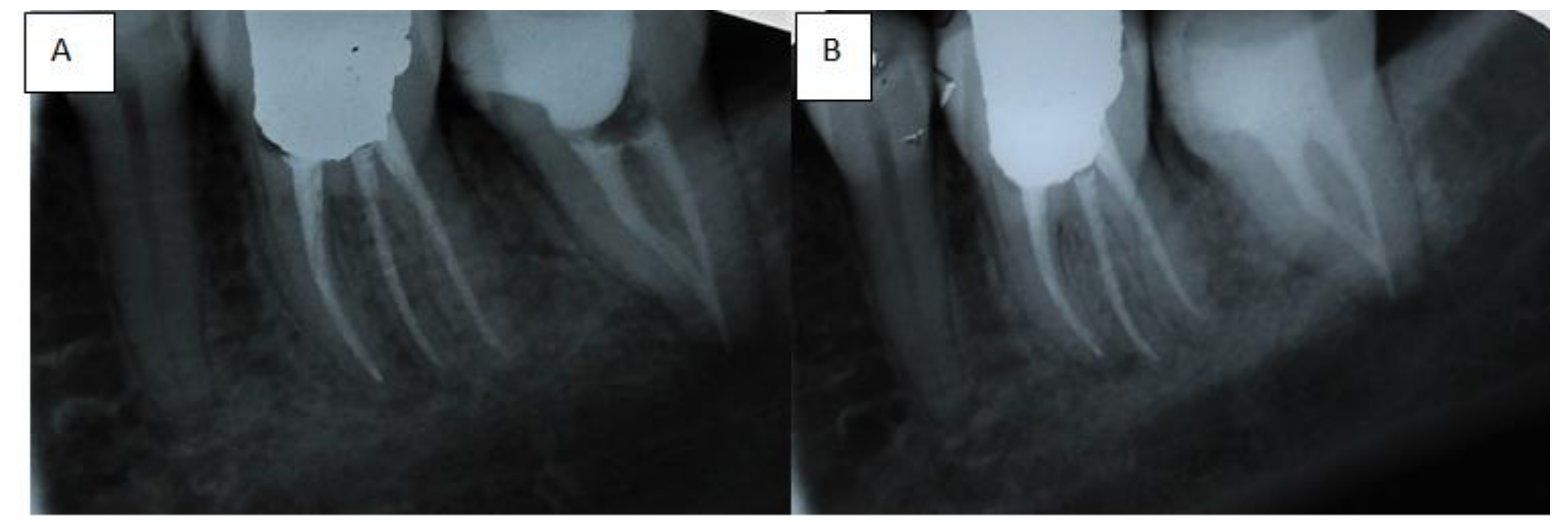

[Figure-3]; a- immediate Postobturation radiograph, b- Postobturation radiograph after 6 months.

\section{Conclusion:}

The present case report discusses the endodontic management of an unusual case of a mandibular second molar with Vertucci type II canal pattern (i.e. two canal orifices join together and exit as one apical foramen). Although the incidence of root variations is rare, their importance should not be underestimated. Careful examination of radiographs and internal anatomy of teeth are essential. The present case confirms the necessity for meticulous examination of the pulpal floor at high magnification under sufficient illumination of the dental operating microscope and highlights the importance of newer imaging techniques like CBCT scanning in preoperative assessment.

\section{Acknowledgements:}

None

\section{References}

[1]. Article O. Root Canal Morphology of Permanent Mandibular Second Molar in a South Indian Population using Computed Tomography. 2016;1(June):1-5.

[2]. Vertucci FJ. Root canal anatomy of the human permanent teeth. Oral Surg Oral Med Oral Pathol [Internet]. 1984 Nov [cited 2016 Sep 18];58(5):589-99. Available from: http://www.ncbi.nlm.nih.gov/pubmed/6595621

[3]. Chokshi S. Morphological variations in the root canal system of mandibular second molar : A case series. :135-8. 
[4]. Baratto-Filho F, Fariniuk LF, Ferreira EL, Pecora JD, Cruz-Filho AM, SouzaNeto MD. Clinical and macroscopic study of maxillary molars with two palatal roots. Int Endod J. 2002; 35(9):796-801.

[5]. Frank j., Vertucci, Root canal morphology and its relationship to endodontic procedures; Endodontic Topics

[6]. 2005, 10, 3-29.

[7]. Kala M., Chandki R., Lodha E., Shaktidar P., c shaped canal configuration: a diagnostic dilemma ; AOSR 2011;1(2):79-83.

[8]. Patel S, Dawood A, Whaites E, Pitt Ford T. New dimensions in endodontic imaging: part 1 -conventional and alternative radiographic systems. Int Endod J 2009;42: 447-62. 